\title{
Rursus
}

Russus

Poiétique, réception et réécriture des textes antiques

$1 \mid 2006$

Le modèle animal (I)

\section{Philomèle : Du mythe aitiologique au début du mythe littéraire}

Michèle Biraud et Evrard Delbey

\section{OpenEdition}

Journals

Édition électronique

URL : http://journals.openedition.org/rursus/45

DOI : $10.4000 /$ rursus.45

ISSN : 1951-669X

Éditeur

Université Nice-Sophia Antipolis

Référence électronique

Michèle Biraud et Evrard Delbey, « Philomèle : Du mythe aitiologique au début du mythe littéraire », Rursus [En ligne], 1 | 2006, mis en ligne le 09 juillet 2006, consulté le 01 mai 2019. URL : http:// journals.openedition.org/rursus/45; DOI : 10.4000/rursus.45

Ce document a été généré automatiquement le 1 mai 2019.

Rursus 


\title{
Philomèle : Du mythe aitiologique au début du mythe littéraire
}

\author{
Michèle Biraud et Evrard Delbey
}

1 Nous allons commencer cette journée d'étude sur le modèle animal par un mythe à fondement animalier, celui de Térée, Procné et Philomèle. Nous espérons parvenir à vous montrer que ce mythe présuppose une observation attentive des interactions entre trois oiseaux dont le comportement animal a été interprété sur le modèle des relations sociales et subjectives entre êtres humains et dans le cadre d'homologies entre langage des oiseaux et langage des hommes. En retour, dans le processus de constitution du mythe, le modèle animal est premier, comme le montrera l'étymologie des noms des personnages et les mots qu'ils prononcent.

2 Chacun sait aussi que dans ce mythe, la relation entre les noms de Procné et Philomèle, les deux rôles d'épouse et de jeune sœur, et les oiseaux de métamorphose, manque étrangement de stabilité. Nous en proposerons une explication elle aussi doublement justifiée par l'ornithologie et l'onomastique, puis nous étudierons les retentissements de cette instabilité dans les textes qui sont postérieurs à ses premières manifestations. Nous verrons que chaque auteur grec ou latin remodélise le mythe selon ses préoccupations, proposant à l'homme les animaux du mythe comme un miroir à multiples facettes.

\section{Les textes grecs}

De nombreux textes grecs évoquent ces personnages, mais il s'agit le plus souvent d'allusions très brèves à une fable supposée connue du lecteur. Les tragédies sur Térée et les Pandionides étant perdues, on ne trouve aucune narration complète de cette fable dans aucun texte de l'époque classique, il faut attendre les mythographes, les lexicographes et les scholiastes ${ }^{1}$. La comparaison des textes d'Apollodore ${ }^{2}$, d'Antonius Libéralis ${ }^{3}$ et du vers 518 du chant 19 de l'Odyssée (et sa scholie) ${ }^{4}$ montre en outre qu'il existe au moins trois histoires distinctes ${ }^{5}$ dont le noyau commun est une reine - rossignol pleurant le fils qu'elle a tué. On a d'une part la légende thébaine de Aédon, fille de Pandaréos, épouse du roi thébain Zéthos, qui tua son fils Itylos par méprise, en voulant 
tuer, par jalousie pour sa belle-sœur, l'un de ses neveux. C'est à cette histoire qu'Homère fait allusion. La fable la plus connue des Anciens, qui a donné lieu au plus grand nombre de variations (voir par exemple les textes d'Eschyle ${ }^{6}$, de Sophocle ${ }^{7}$, d'Euripide ${ }^{8}$, du Rhésos ${ }^{9}$, $d^{\prime}$ 'Aristophane ${ }^{10}$, d'Achille Tatius ${ }^{11}$, de Babrios ${ }^{12}$, de Nonnos ${ }^{13}$, de Libanios ${ }^{14}$ ) et d'allusions, est celle que rapporte Apollodore. Le récit d'Antonius Liberalis comporte plus de personnages de la famille des deux sœurs et se passe dans une région différente: c'est peut-être un essai de synthèse de plusieurs variantes de la fable précédente.

\section{L'état grec du mythe, où Procné, l'épouse, est rossignol, et Philomèle, sa sœur, est hirondelle}

On ne s'intéressera ici qu'à la version de la fable à trois personnages, celle qu'on appelle le mythe mégaro-athénien, et qui raconte les relations entre l'époux, l'épouse et la sœur de l'épouse, le meurtre volontaire d'Itys par sa mère, puis la poursuite des criminelles par le père et la métamorphose de chacun d'eux en oiseau. C'est celle-là qui est au fondement de presque toutes les allusions des textes littéraires grecs et latins.

5 Ce qui est surprenant dans les textes qui la relatent, c'est que la métamorphose puisse se réaliser en des oiseaux différents: Procné, l'épouse, et sa sœur Philomèle, deviennent respectivement, dans les textes grecs, un rossignol et une hirondelle ${ }^{15}$; mais par ailleurs, certains textes latins présentent sans ambiguïté l'équivalence Philomèle - rossignol et Procné - hirondelle, et tous ne nomment pas l'épouse « Procné » et sa sœur « Philomèle ». De plus, Térée est transformé en huppe dans la plupart des récits du mythe, mais on le voit apparaître aussi sous la forme d'un petit rapace (faucon ou épervier). Une inversion des figures de métamorphose, et une double possibilité de métamorphose animale, tout ceci n'est pas coutumier dans les mythes, et n'a jamais été expliqué.

Notre premier objectif est d'apporter des éléments de réponse qui reposent sur le rapport entre données linguistiques (la signification des onomata) et ornithologiques, et s'appuient aussi sur les conceptions que les Anciens avaient du comportement et des cris de ces oiseaux, si peu logiques qu'elles puissent nous paraitre.

\subsection{Procnè et Philomèle}

\subsubsection{Les noms des deux sœurs décrivent les deux oiseaux}

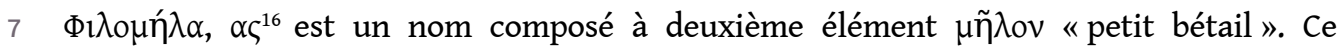
deuxième élément n'est pas rare dans l'onomastique, principalement en Béotie ${ }^{17}$. La signification d'« amie du petit bétail » s'explique par le fait que les hirondelles, oiseaux insectivores, pourchassent les mouches qui abondent au voisinage des troupeaux.

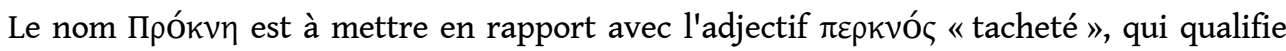
des raisins ou des olives qui mûrissent (et signifie en G. Mod. «au visage couvert de taches de rousseur »). Ces deux mots ayant le suffixe -vóc, -vń, qui permet de créer des dérivés possessifs, ils signifient " pourvu de taches ». La variation de radical est conforme aux différentes formes possibles pour un thème d'origine indo-européenne, et le radical *

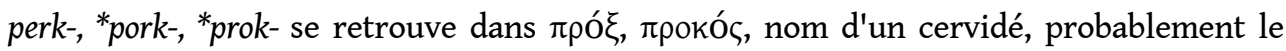

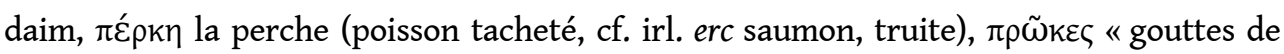
rosée $»^{18}$. Le sens de ce nom qui fut un ancien adjectif est « couvert de taches» et non pas 
《sombre $»^{19}$. La difficulté jusqu'ici non-résolue est que cette qualification ne semble pas pouvoir caractériser le rossignol. Celui que nous connaissons en Europe de l'Ouest a en effet le dos brun-roux et la gorge et le ventre beiges, mais son plumage ne porte aucune tache, aucune variation notable de couleur. C'est aussi ce qui a causé l'embarras des

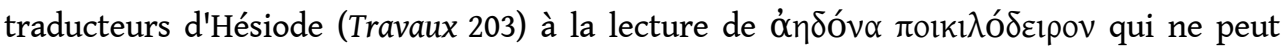

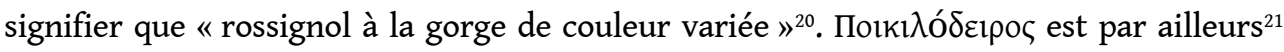
une qualification de la sarcelle : le plumage de sa gorge est beige avec des dessins bruns en forme de petits demi-cercles, comme s'il était taché par des gouttes d'eau trouble. Aucun commentateur des auteurs grecs ne semble s'être avisé qu'il existe plusieurs espèces de rossignols : à côté de celle que nous connaissons, le luscinia megarhynchos, il y a une autre espèce, le luscinia luscinia, qui fréquente la Sibérie occidentale et l'Europe orientale, et qui a, comme la sarcelle, le ventre couvert de petites taches brunes en forme de gouttes ${ }^{22}$.

Le fait que les deux femmes soient désignées par des noms qui ne conviennent qu'aux oiseaux en qui le mythe va les métamorphoser implique que leur état animal a été pensé le premier dans la construction du mythe.

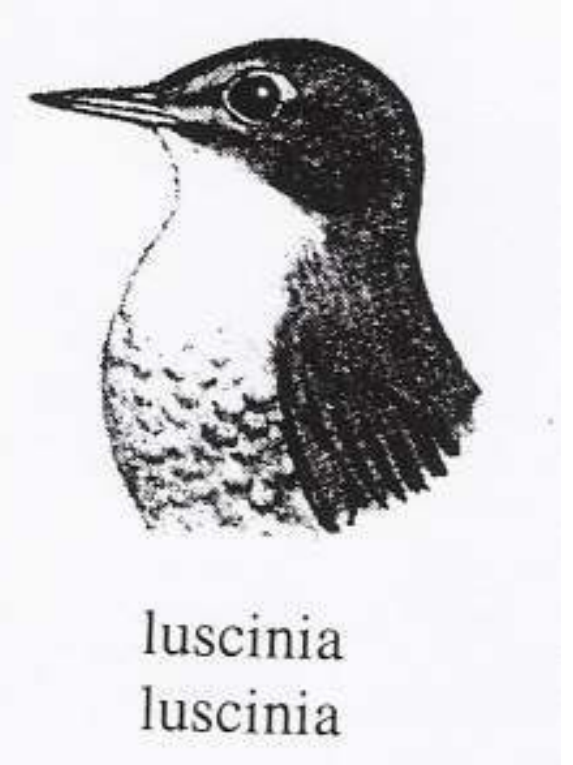

\subsubsection{Leurs cris d'oiseaux interprétés en noms : Itys et Térée}

Le signifié des noms n'est pas le seul indice qu'il s'est agi à l'origine d'une histoire d'animaux. Selon des traditions dont Eustathe et les scholiastes se font l'écho ${ }^{23}$, ces personnages ont continué après leur métamorphose à prononcer chacun un mot du langage humain :

Philomèle s'envole changée en hirondelle et Procné en rossignol. C'est pourquoi l'hirondelle a la voix rauque et un chant désagréable à cause de sa langue coupée et, en bégayant constamment, profère le nom de Térée, et c'est aussi pourquoi le rossignol émet «Itys » dans son chant. Et Térée métamorphosé en huppe ne fait que s'exclamer « où sont-elles? »(Eustathe, Commentarii ad Homeri Odysseam, Vol. 2, p 215) ${ }^{24}$.

Procné, ayant appris les malheurs de sa sœur, égorgea son fils Itys et en fit un repas pour Térée. Lorsque celui-ci comprit, il les poursuivit avec son épée en criant « où? où? ? 


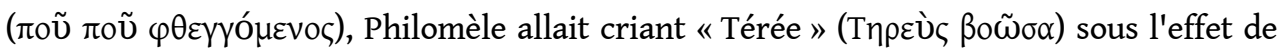
la panique, et Procné, en se lamentant sur Itys ( $\tau$ òv 'I $\tau u v \theta \rho \eta v o \tilde{\sigma} \sigma \alpha$ ), disait plaintivement

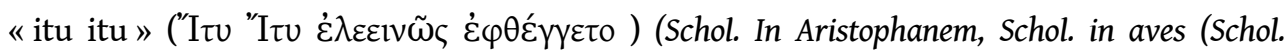
vetera), Argumentum-scholion schav, v 212).

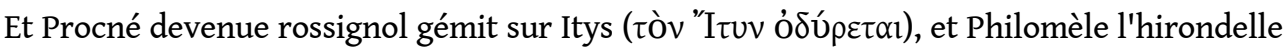

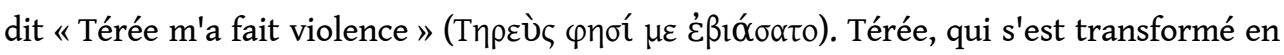

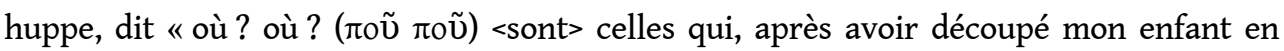
morceaux, me l'ont présenté en festin?». Telles sont les divagations mythologiques. Sophocle a écrit sur ce sujet dans sa tragédie Térée. (Schol. In Hesiodum, Schol. in opera et dies (Schol. vet..., Prolegomenon-scholion sch, p-v 566ter).

14 La croyance en ce genre de phénomène n'est qu'exceptionnellement attestée chez les Grecs, mais Elien en témoigne aussi à propos de perdrix qui auraient rendu un oracle (De natura animalium, XV, 27) : il rapporte une tradition selon laquelle des francolins (attagas) prophétisèrent un jour [tris tois kakois ta kaka] (=«Trois fois des malheurs pour les mauvais ») ou plutôt, avec iotacisme, si l'affaire n'est antérieure que de deux ou trois siècles par rapport à Elien [tris tis kakis ta kaka], et cela « avec une élocution bien plus claire et en articulant bien mieux que les bébés »; ceux qui les entendirent interprétèrent sans doute ainsi des cris phonétiquement proches, le genre de cris d'oiseaux désignés en français par le verbe onomatopéique cacaber. D'autre part, le poète archaïque Alcman semble avoir écrit qu'il devait l'idée du chant humain au chant des perdrix, d'après ce passage d'Athénée (IX, 390 a) :

Les perdrix sont appelées caccabai par certains auteurs, comme Alcman, par exemple lorsqu'il dit : « la poésie épique et lyrique, à voix haute, Alcman l'a inventée en composant les notes de la perdrix $»^{25}$, indiquant ainsi clairement qu'il avait appris à chanter grâce aux perdrix ${ }^{26}$.

Des cris d'oiseaux pouvaient donc être interprétés comme des mots de la langue grecque, et leurs chants comme analogues au chant humain. Mais, dans le mythe de Térée, quels cris sont au départ de ce type d'assimilation? On va évidemment raisonner à partir des onomatopées par lesquelles les Grecs rendaient ces cris, lorsqu'elles nous sont accessibles directement ou par le verbe qui en est le dérivé.

17 Pour le cri de l'hirondelle, il existe deux verbes onomatopéiques en grec, tous deux signalés dans le Lexique d'Hésychius: le verbe $\tau \varepsilon \rho \varepsilon \tau i ́ \zeta \varepsilon i{ }^{27}$, à associer au nom de même radical $\tau \varepsilon \rho \varepsilon ́ \tau l \sigma \mu \alpha$, et le verbe $\tau 1(\tau) \tau u \beta i ́ \zeta \varepsilon ı v^{28}$. Bien qu'aucun texte grec ne l'explicite, ces deux verbes sont la transcription onomatopéique de cris d'espèces différentes: si toutes les espèces vocalisent en [i], il n'y a que dans le cri de l'hirundo rustica qu'apparaissent des sons [ou] et [u], qui correspondent au upsilon de $\tau 1(\tau) \tau \cup \beta i ́ \zeta \varepsilon v v$, alors que $\tau \varepsilon \rho \varepsilon \tau i ́ \zeta \varepsilon l v$ est une onomatopée assez proche du cri de l'hirondelle de rocher (hirundo rupestris) [trrit tschrrrip]. C'est d'ailleurs probablement de l'hirondelle de rocher qu'il est question dans le mythe: c'était alors l'espèce la plus proche des troupeaux puisque le troupeau du Cyclope, et sans doute celui de plus d'un berger, avait son gite dans une grotte.

On peut rapprocher l'indication du mythe que Philomèle crie "Térée» du verbe

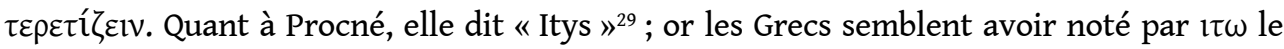
cri qui entrecoupe le chant du rossignol, si l'on en juge par un fragment papyrologique de tragédie ${ }^{30}$. 


\subsection{Térée}

\subsubsection{Huppe et épervier (kirkos)}

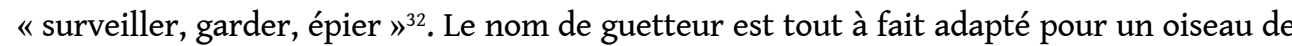
proie comme l'épervier : c'est son activité principale. Pour la huppe, la seule façon de justifier ce nom est de le relier à son cri soudain qui est interprété par tous les animaux $\mathrm{du}$ voisinage comme un cri d'alerte, qui rend tout le bois silencieux à l'écoute des indices pour identifier le prédateur qui a suscité ce cri. Toutefois le houpoupou de la huppe n'est pas réellement un cri d'alarme, c'est un cri que l'on n'entend qu'à la période de la formation du couple et de la nidification ${ }^{33}$.

\subsubsection{Le cri de Térée transformé en oiseau}

Le cri que le mythe prête à Térée ( $\pi$ oṽ ...) est une très bonne notation du cri de la huppe, qui émet ordinairement un cri trisyllabique poupoupou. Et pour les Grecs, dans leur langue, ce cri avait un sens : $\pi$ oṽ ... « où "sont-elles" ? » ${ }^{34}$. Cette question est éminemment adaptée à un guetteur.

Il faut noter que l'interprétation du cri [poupoupou] comme une triple occurrence de l'adverbe interrogatif de lieu n'est possible que dans certains dialectes du grec (langue homérique, attique $)^{35}$. Avant l'époque mycénienne, la prononciation de cet adverbe était [ $\left.\mathrm{k}^{\mathrm{w}} \mathrm{ou}\right]$. Or, en émission doublée, c'est à peu près le cri du coucou. Si le mythe est antérieur à l'époque mycénienne, et si l'interprétation des cris des oiseaux est constitutive du mythe, Térée aurait pu être au départ un coucou, déguisé ensuite en huppe à cause de l'évolution phonétique de l'adverbe de lieu qui était l'interprétation obligée de son cri.

Ce remplacement du coucou par la huppe après l'évolution de l'occlusive à appendice labio-vélaire montre chez son auteur la permanence de la conception que certains cris 
des oiseaux sont des mots de la langue des humains, conception que nous avons vue par ailleurs à propos des oiseaux qui incarnent les deux sœurs.

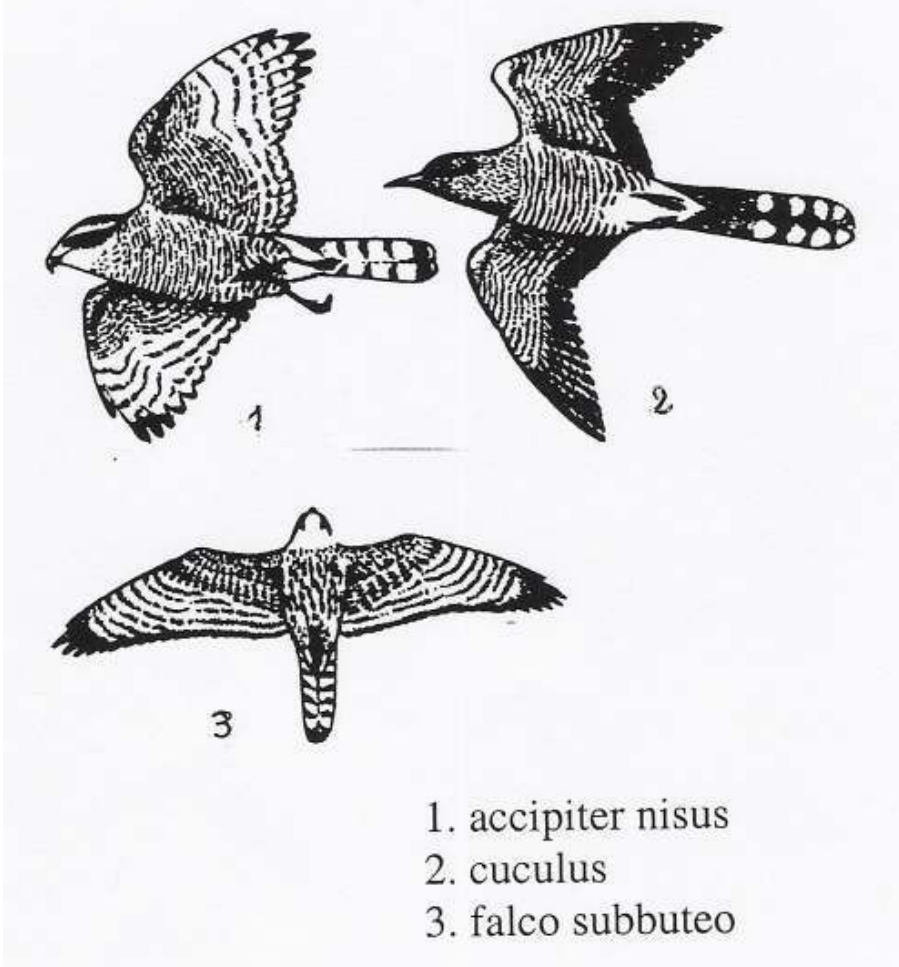

\subsubsection{Un coucou qui est aussi un petit rapace?} petits rapaces prédateurs de petits oiseaux, l'accipiter nisus (ou épervier ${ }^{40}$ ) et le falco subbuteo (ou faucon passetier ${ }^{41}$ ou hobereau) par leur taille et leur envergure ${ }^{42}$, leur forme générale en vol, et aussi par la couleur grise de leur plumage. Les ouvrages d'ornithologie modernes mettent d'ailleurs en garde contre cette possibilité de confusion. Tout en rangeant le coucou parmi les hierax, Aristote soulignait toutefois déjà des différences : 

mieux que celui de la huppe la poursuite finale du mythe que se font les protagonistes métamorphosés en oiseaux. Si belliqueuse que puisse paraître la huppe, avec sa houppe déployée qui évoque une aigrette de casque, et son long bec, qu'elle peut sembler brandir comme une épée, elle n'est qu'un insectivore. En revanche, la nourriture du plus petit des éperviers et du faucon hobereau est presque entièrement composée de petits oiseaux qu'ils attrapent en vol ou perchés sur une branche. Le faucon hobereau est particulièrement friand d'hirondelles, et la fable rapportée par Hésiode (Travaux, 202-212) montre un hierax avec un rossignol dans ses serres. La phase finale du mythe, où Térée se mue en oiseau de proie poursuivant le rossignol et l'hirondelle, aurait donc été inspirée par l'observation de la nature. coucou. En effet, parmi les autres oiseaux où les couples sont au moins aussi durables que le temps de la couvaison, de la nourriture et de l'éducation des oiselets, les coucous sont une exception scandaleuse. Pendant la période de reproduction, il ne se forme pas un couple parental : la femelle s'accouple successivement avec plusieurs mâles (et bien sûr 
les mâles ont tout aussi « infidèles ») ; elle pond environ dix œufs à l'intervalle d'au moins deux jours et dépose son œuf dans le nid d'un oiseau insectivore, qui couvera et nourrira son petit à sa place. Les Grecs n'ignoraient pas cela, comme en témoignent Aristote (H.A. 618a, 8-30) et Elien (N. A. III, 30). La bigamie de Térée serait donc analogue à la polygamie du coucou.

Le personnage de Térée associerait donc tout l'égoïsme et la liberté sexuelle du coucou avec toute la férocité du rapace. Ces deux oiseaux sont un bien meilleur modèle animal pour un homme adultère et cruel que la huppe, oiseau paisible, bon père de famille et qui aime la solitude.

La situation de parasitisme du nid peut éclairer aussi la réaction finale de la mère d'Itys. Elle serait en effet un rossignol qui se serait trouvée mère d'un petit coucou. Il n'est évidemment pas dans la nature d'un oiseau de tuer ses propres enfants, mais un oiseau pourrait vouloir tuer le fils de coucou qu'il a nourri au moment où il s'aperçoit de sa vraie nature de coucou. Elien (N.A. III, 30) signale la possibilité de mauvais traitements :

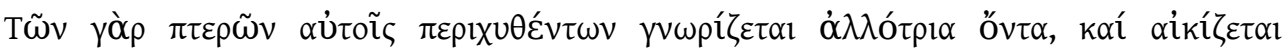
$\pi ı \kappa \rho o ́ \tau \alpha \tau \alpha$.

[si les petits coucous n'ont pas quitté le nid auparavant], lorsqu'ils ont tout leur plumage il devient évident qu'ils sont d'une autre espèce [que leurs parents nourriciers] et ils ont à subir les traitements les plus cruels.

Que ces mauvais traitements soient effectifs ou seulement imaginés, les Grecs les concevaient comme plausibles, et cette observation fournit une clé du comportement infanticide de Procné en tant que rossignol.

\subsubsection{Des cris d'oiseaux aux voix humaines : une homologie}

Un autre facteur important dans la création du mythe est l'assimilation des cris d'oiseaux aux différentes formes de l'expression langagière des hommes.

Dans le mythe, la huppe et le coucou ont pu être assimilés à un homme à cause du timbre grave de leur cri, tandis que les tonalités plus aiguës du rossignol ${ }^{43}$ et de l'hirondelle ont été assimilées à des voix féminines. La même répartition dans le genre grammatical des noms de ces oiseaux a dû procéder aussi de la même assimilation dans un certain nombre de langues ${ }^{44}$.

Par ailleurs, les Anciens ressentaient la tonalité du chant du rossignol comme plaintive. Beaucoup de textes, notamment des tragédies, en témoignent ${ }^{45}$. $Y$ abondent les notions de

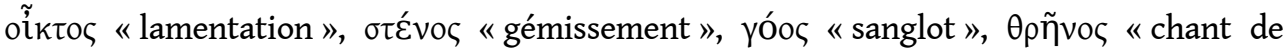
deuil », et les occurrences du verbe ỏ psychologique a dû amener à se demander pourquoi ce thrêne perpétuel, nuit et jour, pendant un mois au printemps. Or quel plus grand malheur pour une femme que la mort de son fils, surtout si elle en est cause?

Parmi les espèces d'hirondelles dont le cri correspond à l'onomatopée dont dérive le verbe

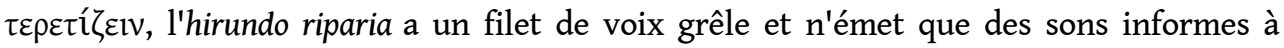
dominante de [pfii], l'hirundo rupestris émet un cri plus ferme mais tout aussi inarticulé (à peu près [tchripp]). On comprend que les Anciens aient rapproché la voix de l'hirondelle de celle d'un bègue ${ }^{46}$, ou aient pu assimiler son expression à celle d'un étranger, d'un barbare. Ainsi, d'après Hérodien, Eschyle a forgé sur le nom de l'oiseau un verbe délocutif $\chi \eta \lambda_{1} \delta o v i ́ \zeta \varepsilon ı v$ signifiant $"$ parler comme un barbare ${ }^{47}$, et Clytemnestre assimile aussi le 
langage de l'étranger à celui de l'hirondelle (Esch. Agam. 1050) ${ }^{48}$. La Philomèle muette de la fable, qui est plutôt une semi-muette puisqu'elle a su parler, qu'elle peut émettre certains sons et qu'il lui manque seulement la langue pour articuler certains phonèmes, émet, comme ces hirondelles, des sons inarticulés, un babil inintelligible.

La confrontation des voix de ces trois oiseaux correspond à une tripartition des comportements vocaux des êtres humains : le cri articulé et syllabé, le chant (qui traite les syllabes en vocalises modulées), le bredouillis inarticulé. Cette partition langage articulé / bredouillis / chant est celle-là même dont use Aristote pour classifier la parole des animaux à partir du modèle humain de langage (H.A. I, 1, 488a); il y ajoute l'opposition bavard / taciturne, importante aussi dans l'affectation des rôles aux personnages du mythe :

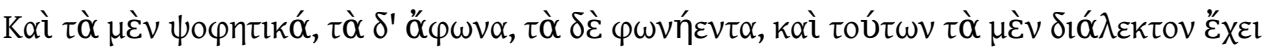

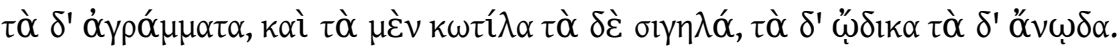

Certains animaux émettent des sons, d'autres sont muets, d'autres possèdent une voix; parmi ces derniers, les uns ont un langage articulé, les autres non. Les uns sont bavards, les autres taciturnes ; les uns sont des chanteurs, les autres non.

Le choix de ces trois oiseaux s'avère donc prototypique des partitions du langage humain.

\subsubsection{Un mythe qui modélise les rapports de force et de langage entre l'homme et les femmes}

Le choix de ces trois oiseaux répond aussi, dans la logique du mythe, à une modélisation des rapports entre homme et femmes dans la distribution sociale de la parole. Si taciturne que soit le chef de famille, il parle le langage humain, et son épouse aussi (le rossignol émet des syllabes dans son chant car on peut interpréter ainsi ses [tio tio tio ito ito]), la part privilégiée d'expression publique de l'épouse étant les cris et chants de déploration. En revanche, la fille non mariée est socialement privée de parole en dehors du cercle familial, et si elle subit des violences, elle ne verra pas sa parole écoutée sous peine de déshonneur, donc d'un retranchement social encore plus grand. Elle est réduite au silence, ou à des cris gémissants et un babil confus que l'on ne peut interpréter. Ce mythe permet de penser et d'exprimer métaphoriquement ces rapports.

\subsection{Conclusion sur l'état du mythe que présentent les textes grecs}

51 On peut donc affirmer, au terme de cette étude, que les trois personnages mythiques ont, dès l'état le plus ancien du mythe, des noms signifiants en fonction des caractéristiques des oiseaux qui sont leur métamorphose finale. Et ces animaux ont des comportements qui, une fois interprétés et évalués selon les usages et la morale des hommes, sont au fondement du comportement des héros. Les cris de ces animaux sont représentatifs, par leurs caractéristiques sonores, des partitions du langage humain. Ils sont aussi interprétés en mots humains, les noms de Térée et de son fils Itys. Donc dans la phase d'observation des animaux, des cadres mentaux aptes à décrire des comportements humains ont été utilisés comme grille de lecture des comportements animaux, puis dans la phase de création du mythe, la transformation s'est faite d'oiseaux en hommes. L'animal a alors servi de modèle à l'homme. semble avoir été le déclencheur qui a fait d'une fable animalière un vrai mythe. L'histoire 
d'adultère et de vengeance n'a de fondement animalier que compte tenu des mœurs du coucou; après la substitution, cette histoire n'est plus motivée que dans le domaine humain. La huppe a quelque chose de solaire et de royal dans son apparence (et qui est actualisé dans les mythes égyptiens et persans), alors que personne n'aurait l'idée de faire du coucou un roi parmi les oiseaux. Térée est alors devenu un roi aux mœurs violentes. Le modèle humain a alors pris le dessus, et les personnages ne sont restés des animaux que par leur métamorphose finale ${ }^{49}$.

\section{La réception de ce mythe dans la littérature latine}

\section{1. États du mythe dans la poésie latine de Catulle à Martial}

À regarder maintenant quelques textes latins, nous constatons des interversions dans les rôles. Notre « corpus » d'auteurs latins prend en compte Virgile, les poètes élégiaques de Catulle à Ovide, ainsi que Martial. Assurément il est limité, mais il nous est déjà apparu fort instructif quant aux significations des deux figures féminines de Procné et Philomèle.

Si nous commençons par les occurrences des métamorphoses certaines, nous trouvons d'abord Virgile. Dans les Géorgiques, IV, 15, il est question de Procné qui porte, marquée sur sa poitrine, l'empreinte de ses mains ensanglantées ${ }^{50}$. Notons d'abord qu'ici il s'agit d'une autre espèce d'hirondelle que celle du mythe grec, celle-ci a une tache rouge sous le bec, c'est l'hirundo rustica. Dans ce texte, Procné est épouse et mère et c'est l'hirondelle. Il s'agit d'installer un rucher et d'éloigner les oiseaux insectivores; le poète insiste sur la trace du crime ancien et donc sur le danger que représente l'hirondelle.

Puis nous trouvons chez Ovide, dans l'Art d'aimer, II, 383-384 qui plaide pour le secret des infidélités dans les relations adultères ${ }^{51}$, Procné comme exemple à éviter: si Procné n'avait pas su l'adultère de Térée, elle n'aurait jamais commis le pire.

Le même auteur, dans les Fastes, II, 627-630 $0^{52}$, puis II, 853-856 $6^{53}$, rappelle la mère criminelle que fut Procné en tant qu'épouse de Térée. Quant au passage des Tristes, III, 12, 9-10, qui évoque l'arrivée du printemps en Italie, alors qu'au pays des Gètes l'hiver se prolonge ${ }^{54}$, il insiste encore sur le crime commis par l'hirondelle.

Comme autre référence certaine, nous avons chez Martial, Épigrammes, XIV, 75, la métamorphose de Philomèle, sœur de l'épouse, en rossignol ${ }^{55}$.

Enfin, nous rencontrons sous cette même forme du rossignol Philomèle épouse et mère dans la Bucolique VI, $78-81^{56}$ où Silène chante diverses métamorphoses; même si l'allusion au toit de la demeure évoque l'hirondelle, les deserta font songer aux espaces solitaires des profondes forêts où le rossignol désormais habite. De même, dans les Géorgiques, IV, 511-515 ${ }^{57}$, le poète représente Orphée qui regrette son épouse définitivement perdue et devient comparable à Philomèle pleurant ses petits dont un durus arator l'a privée. La référence est générique et ne s'inscrit pas directement dans le mythe d'Itys: le rossignol représente la déploration maternelle en soi ; toutefois, il est logique de penser que le poète se réfère à la version où Philomèle, mère d'Itys, est un rossignol. Ovide, quant à lui, dans l'élégie II, 6, 7-10 des Amours ${ }^{58}$ sur la mort du perroquet de Corinne, rappelle les pleurs de Philomèle rossignol à la mort de son fils Itys. En même temps, le poète dédramatise la référence en incitant Philomèle à gémir sur une disparition plus récente, celle du perroquet de Corinne précisément. Cette désinvolture à propos des plaintes maternelles et du crime de Térée ne serait-elle pas propice à 
l'écriture d'un texte-palimpseste où Procné et Philomèle échangeraient leurs fonctions et leurs métamorphoses, à l'écart de toute version "officielle ", définitive, de la légende ? Ne sommes-nous pas à la fois dans le mythe et la fecunda licentia uatum qui se déploie dans l'illusion mensongère (III, 12, 41-44)?

On peut alors comprendre que F. Létoublon ait souligné la structure complexe du mythe de Procné et de Philomèle. Dans le numéro 904-905 (août-septembre 2004) de la revue Europe intitulé «Mythe et mythologie dans l'antiquité gréco-romaine», F. Létoublon a écrit, en effet, un article intitulé « Le rossignol, l'hirondelle et l'araignée » (p. 73 à 102), qui est consacré précisément au mythe de Procné et de Philomèle et à celui d'Arachné. Partant des Métamorphoses d'Ovide, elle essaie de montrer que les personnages de Procné et de Philomèle, qui nous intéressent présentement, sont issus du «grand récit anonyme de la mythologie grecque où Ovide a puisé son inspiration » (p. 73).

Nous reprendrons d'abord l'analyse que notre collègue fait de l'étiologie mythique du rossignol telle que nous la livre le récit du livre VI des Métamorphoses, du vers 412 à 674 . «Ovide nomme explicitement tous les personnages de cette légende : le roi d'Athènes, Pandion, admirateur des exploits guerriers du roi thrace Térée, lui donna en mariage sa fille Procné, qui devait donner naissance en Thrace à un fils appelé Itys. Cinq ans plus tard, Procné -ironie tragique - supplie son mari de lui permettre de revoir sa sœur Philomèle. Sans emmener Procné avec lui, Térée retourne à Athènes demander à Pandion sa deuxième fille, dont il tombe immédiatement amoureux. Pandion, non sans difficulté, confie Philomèle à Térée qui l'emmène en Thrace et, à peine abordé sur les rivages, l'enferme dans une bergerie isolée au milieu d'une épaisse forêt et la viole. Elle répond par de terribles imprécations et menace de tout raconter à sa sœur, demandant la mort. Térée lui coupe sauvagement la langue et retourne auprès de Procné qui réclame sa sœur. Il lui fait un faux récit de la mort de Philomèle et celle-ci lui célèbre des funérailles près d'un tombeau vide. Cependant Philomèle se fabrique un métier à tisser, stamina barbarica, grâce auquel elle tisse un message pour sa sœur (en lettres de pourpre sur fond blanc, purpureasque notas filis intexuit albis, image du sang sur la pureté virginale), qu'une servante porte à sa maîtresse. En lisant l'inscription, Procné garde le silence et ne pense plus qu'à la vengeance. Pendant la nuit, elle devient bacchante et ramène sa sœur au palais. A l'arrivée de son enfant Itys, elle s'attendrit mais la folie de la vengeance la reprend, elle frappe Itys d'une épée et Philomèle ajoute ses coups à ceux de sa sœur. Elles mettent le corps de l'enfant en pièces et le font bouillir dans un chaudron, puis elles apportent ce repas à Térée, qui réclame Itys. Procné lui révèle la vérité dans une phraseénigme (655 intus habes, quem poscis, «tu as à l'intérieur de toi celui que tu réclames »), et Philomèle brandit la tête de l'enfant (...). Tandis que Térée essaie de poursuivre les deux soeurs, elles sont déjà suspendues dans les airs, transformées en oiseaux, sans qu'Ovide précise lesquels. < c'est nous qui mettons en italiques >. Térée, lui, est transformé en huppe, dont "la tête a l'air armée », facies armata uidetur (p. 74-75). Après avoir rappelé l'insistance avec laquelle Ovide décrit la barbarie de Térée, F. Létoublon remarque, avec raison, que cette barbarie même s'étend aux deux sœurs: «La fureur de Procné à la lecture du message tissé par sa sœur la transforme en bacchante et lui fait entraîner Philomèle dans le délire bachique. Au moment où elle va porter l'épée sur son fils, Procné est comparée à une 'tigresse des bords du Gange' (636-637); la description de la préparation du repas et de la tête d'Itys brandie par Philomèle sont dans le même style...» (p. 76). Enfin, et surtout, à propos de la double métamorphose de Procné et de Philomèle, F. Létoublon écrit: «Très détaillé sur les actes de sauvagerie des deux côtés, le récit est en revanche 
étrangement allusif sur la métamorphose et l'explication qu'elle permet: toute la tradition semble faire du chant du rossignol la raison principale de toute l'aventure, mais Ovide n'en dit rien, il faut que nous bâtissions l'explication nous-mêmes (...) Quant à l'hirondelle, plusieurs des allusions d'Ovide aux cris inarticulés de Philomèle une fois qu'elle a la langue coupée suggèrent qu'une des fonctions du mythe est d'expliquer les cris rauques et peu mélodieux de l'hirondelle par opposition à la musique naturelle du rossignol» (p. 76-77). Si nous comprenons bien l'argumentation de F. Létoublon, le nom propre de Philomèle signifiant « qui se plait au chant, qui aime le chant » conviendrait mieux à Procné transformée en rossignol qu'à sa sœur "transformée en hirondelle justement parce qu'elle a eu la langue coupée et ne peut plus ni parler ni chanter » (p. 77). Par réinterprétation, Virgile lui-même, et Ovide déjà dans les Amours, II, 6, 7-10, ont pu donc faire de Philomèle la mère - épouse métamorphosée en oiseau-chanteur.

\subsection{Justifications ornithologiques et linguistiques de ces altérations du mythe grec}

61 Ces nouvelles configurations d'association entre nom, statut social et oiseau sont en partie motivées à la fois par de nouvelles équivalences entre femme et animal avec d'autres sous-espèces de rossignol et d'hirondelle, au plumage et au cri différents, et par la réinterprétation des noms des deux sœurs.

En effet, le surnom de "tachetée » ne convenait pas au rossignol d'Europe occidentale. Dans ces contrées, Procné ne pouvait donc pas être un rossignol si son nom grec restait intelligible. En revanche, l'hirundo rustica porte une large tache rouge sous le bec, qui a pu être interprétée comme la trace sanglante du forfait de la mère infanticide. La mention de cette tache est récurrente chez les auteurs latins au point que «Procné » peut constituer la désignation générique de l'hirundo rustica. L'hirundo rustica que devient Procné dans ce second état du mythe émet par ailleurs un cri qui a inspiré la création du verbe grec onomatopéique $\tau \imath(\tau) \tau u \beta i ́ \zeta \varepsilon l v$, dans lequel on peut reconnaître, avec un peu d'imagination, le nom d'Itys, car il comporte les phonèmes [itu] dans le bon ordre.

63 Ailleurs, l'allusion inclut l'assimilation entre la mère dénaturée et l'hirondelle, mais sans désigner le personnage par son nom (Ovide, Art d'aimer II, 383-384). Quelquefois, la contiguïté des allusions invite même à voir le nom de Philomèle associé à ce personnage (Virg. Buc. VI, 78-81). Cette confusion des anthroponymes découle sans doute du maintien parallèle de la tradition grecque où Philomèle est l'hirondelle.

Parallèlement à la transformation de Procné en hirondelle à gorge rouge, Philomèle a pu être métamorphosée en rossignol à la suite d'une nouvelle interprétation de son nom. Le rossignol est le plus apte des oiseaux européens à faire des variations dans sa phrase musicale, à l'infléchir, à la prolonger ${ }^{59}$. De ce fait, le nom « Philomèle » a été réinterprété

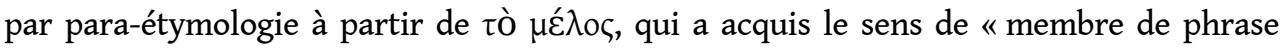
musicale » ou de «chant rythmé » dès le $V^{e}$ siècle ${ }^{60}$. L'épithète $d^{\prime}$ ' amie des chants » est emblématique pour un rossignol. Avec de telles connotations, ce nom s'applique non seulement à la jeune fille violée qui chante son chagrin d'avoir été violentée (Martial, Epigr. Xenia XIV 75), mais aussi à la mère qui chante le deuil de son fils (Ov. Am. II 6, 710, Schol. à Oppien, Eustathe).

Donc, même après l'altération du mythe que constitue l'échange des deux rôles féminins, les noms des deux femmes restent donc motivés en fonction de certaines caractéristiques 
des oiseaux en qui on les métamorphose, soit le plumage, soit le chant, mais pas toujours les deux ensemble.

Les modifications du couple sororal (femmes \& oiseaux) dans les nouveaux états du mythe :

\begin{tabular}{|l|l|l|l|}
\hline & $1^{\circ}$ état & $2^{\circ}$ état & $3^{\circ}$ état \\
\hline & & & \\
\hline épouse et mère & Procné & Procné & Philomèle \\
\hline & la tachetée & la tachée & l'amie des chants \\
\hline & luscinia luscinia & hirundo rustica & luscinia \\
\hline & [itu itu itu] & [tsiuit tuitt] & « Itys » \\
\hline \hline sœur & itw & titubi/zein & \\
\hline & Philomele & Philomele & Procné \\
\hline \hline & l'amie des troupeaux & l'amie des chants & \\
\hline & hirundo rupestris & luscinia & irundo \\
\hline & tereti/zein [tere] & megarynchos & [tere] \\
\hline
\end{tabular}

\subsection{Textes allusifs ou implicites}

66 L'incohérence entre le mythe hérité et ses transformations fait que de nombreux textes latins sont allusifs ou implicites. Une imprécision certaine, tout d'abord, se rencontre dans les Amours, II, 14, 29-3261. Le poète s'en prend à Corinne qui a voulu avorter ; il utilise les exemples de Médée et de la mère d'Itys pour déclarer que toutes deux eurent de graves motifs pour en venir à l'infanticide ; elles avaient à se venger d'un époux, alors que Corinne n'avait aucune raison de tuer l'enfant qu'elle portait. L'épouse de Térée n'est pas désignée nommément, il n'est pas question de sa métamorphose; seule la caractérise l'expression saeua parens qu'elle partage avec Médée (31). En III, 12, 32 ${ }^{62}$, Ovide énumère plusieurs affabulations poétiques parmi lesquelles l'oiseau athénien qui chante l'Odrysien Itys ; même si personnellement nous estimons - comme nous le verrons par la suite - que l'absence de précision suggère la référence à la version sophocléenne, le vers peut se rapporter tout autant à Philomèle qu'à Procné, toutes deux étant filles de Pandion qui est lui-même roi d'Athènes.

67 Catulle, dans le carmen 65 sur la mort de son frère ${ }^{63}$, avait déjà procédé de manière allusive : il s'engageait à composer des chants en l'honneur du défunt, semblables aux gémissements de la Daulienne déplorant Itylos (9-14) ; le poète suivait la version grecque de la légende, mêlant le mythe attique avec une légende thébaine selon laquelle l'enfant s'appelle Itylos (et non Itys) et où il est le fils de Zéthos et d'Aédon (le rossignol); 
l'histoire est différente - la mère tue son fils par erreur, cf. Odyssée, XIX, 518-523 -, mais la contamination fut probablement rendue possible par la proximité des deux noms Itylos/ Itys.

Quant à Properce, il avait évoqué un adunaton à la mesure de la journée merveilleuse qu'annonçait l'anniversaire de Cynthie: que la mère d'Itys s'arrête de gémir en cette occasion (III, 10, 10) $)^{64}$ ! Bien que nulle précision ne figure dans le vers, nous pensons, et nous nous en justifierons bientôt, que c'est la version grecque qui est ici encore adoptée.

Dans le même esprit selon nous, l'Héroïde XV de Sappho à Phaon ${ }^{65}$ semble opter, elle aussi, pour la version sophocléenne: Sappho abandonnée chante comme l'oiseau de Daulis - Procné - déplore la perte de son fils ; uirum non ulta pie maestissima mater (153), le rossignol peuple la forêt silencieuse de sa mélodie (153-156).

70 Mais les Fastes, IV, 481-484 (Cérès pleure la disparition de sa fille, telle la mère d'Itys pleurant la mort de son fill ${ }^{66}$ ) et les Tristes, II, 389-39067 redeviennent imprécis ; là encore, nous serions tenté par l'interprétation selon la version de Sophocle; de même dans les Tristes V, 1, 59-60 ${ }^{68}$, où Ovide allège par sa poésie sa tristitia, à l'image de Procné. Quant aux Pontiques, III, 1, 119-12069 qui s'adressent à l'épouse du poète, ils la conjurent de ne pas renoncer à apitoyer le Prince qui n'est pas l'inpia Progne; nulle précision sur la métamorphose.

71 C'est dire que nous ne pouvons avoir l'ambition de résoudre l'extrême polymorphisme d'un mythe qui n'en est pas pour autant entièrement insaisissable ; c'est pourquoi nous proposerons quelques principes de son utilisation de Catulle à Ovide. Nous commencerons par revenir sur la raison qui nous conduit à supposer le choix de la version sophocléenne, alors que le texte latin est imprécis; puis nous étudierons les caractérisations de Procné et de Philomèle selon leurs fonctions respectives.

\subsection{Usages du mythe dans la poésie latine de Catulle à Ovide}

Si nous procédons par un regroupement d'occurrences, il nous semble possible d'établir que lorsque Procné n'est pas nommée, le poète utilise la version grecque qui est suggérée par des expressions désignant elles-mêmes le contexte grec. Dans les Amours, II, 14, 30, la formule indiquant qu'il s'agit de la mère d'Itys se suffit à elle-même : il n'y a pas lieu de préciser qu'il est question de Procné, puisque la version habituelle de la légende est reprise ; de même en III, 12, 32, lorsque le poète parle du Cecropis ales, dans l'Héroïde XV, 154, lorsqu'il est fait mention du Daulias ales, et dans les Tristes, II, 390, où nous retrouvons la désignation par la périphrase de la mère d'Itys. Ainsi Ovide évoque Procné sous ses trois aspects : la mère, la fille du roi d'Athènes et l'épouse de Térée, roi de Daulis. Le chant douloureux de Procné - rossignol est évoqué soit par le verbe concinit soit par le verbe luget. Catulle et Properce, quant à eux, avaient respectivement parlé de la Daulias absumpti fata gemens Itylei $(\mathrm{c} .65,14)$ et de la mère d'Itys (III, 10,10) pour désigner la même Procné.

Comme chez les Grecs, et ce dès Catulle, nous avons chez les poètes latins la mise en valeur du chant élégiaque, Procné - rossignol étant un marqueur de la tristitia (cf. Héroïde $\mathrm{XV}$ et Tristes , V, 1, 59-60). A noter que, chez Euripide, le terme élégos, voisin d'élégéia (élégie), désigne un chant de deuil et, par analogie, le chant nocturne du rossignol ou le cri de l'alcyon. Le topos de l'amour maternel endeuillé se confond alors avec un genre littéraire particulier et novateur dans la littérature latine. 

qui a pour effet de privilégier la métamorphose de celle-ci en hirondelle, comme si le symbolisme du chant du rossignol devait rester intact. De fait, seul le Chœur des Danaïdes, dans les Suppliantes d'Eschyle, dit la voix du rossignol, pitoyable en ses remords au souvenir du courroux d'une « mère dénaturée » qu'il fut jadis (57-71) et, même en ce cas, la part de beauté dans cette douleur n'est pas oubliée. Au contraire Ovide multiplie les jugements moraux à l'encontre de Procné - hirondelle dans la perspective du livre VI des Métamorphoses qui insiste sur le crime accompli par les deux sœurs et prend soin de ne pas substituer la pitié à la terreur engendrée par la mémoire de l'infanticide et du festin cannibale.

Ovide narrant le mythe insiste fortement, en effet, sur les aspects criminels de celui-ci : au moment même du mariage entre Térée, le Thrace sauveur d'Athènes, et Procné, il montre un hibou sinistre venant se poser sur le toit de la chambre nuptiale (VI, 431-432: profanus bubo); dès l'arrivée de Térée venu chercher Philomèle chez Pandion, nous sommes dans le crimen (472-474) et l'adjectif impius qualifie le roi dénaturé (481-482); nous évoluons dans un univers connoté par le deuil et la plainte sinistre où le désastre est annoncé pour les deux sœurs (485: lugubre). De fait, les cris lamentables de Philomèle violée, lugenti similis, clament vengeance pour le forfait accompli et l'outrage fait aux lois divines et humaines (531-548). Lorsque Procné apprend ce qui s'est passé, elle se situe immédiatement au-delà du bien et du mal, incarnant l'esprit de vengeance (585-586 : (...) sed fasque nefasque / Confusura ruit poenaeque in imagine tota est). A la vue d'Itys, Triste parat facinus tacitaque exaestuat ira (623) et la scène tragique se résume dans la formule que l'épouse s'adresse à elle-même pour s'exhorter au crime-châtiment : (...) scelus est pietas in coniuge Tereo (635). Philomèle, stimulée par sa sœur, s'associe pleinement à l'infanticide et partage avec joie l'accomplissement de cette furialis caedes (636-660). Pour ce qui est de la double métamorphose des sœurs en oiseaux, nous ne pensons pas, contrairement à $\mathrm{F}$. Létoublon, qu'Ovide ait été à ce point allusif : il évoque d'abord la métamorphose de Philomèle en rossignol puis celle de Procné, la mère meurtrière, en hirondelle, constituant ainsi la version latine de la légende : (...) Quarum petit altera siluas, / Altera tecta subit neque adhuc de pectore caedis / Excessere notae signataque sanguine pluma est. (668-670). Certes, René Char que cite notre collègue, parle du «chant d'égorgeur » du rossignol et les poètes grecs peuvent parler aussi du «rossignol fauve» (Esch. Agam. 1140-1149) ou « au bec fauve » (Eur. Hél. 1107-1116), Euripide évoque même son « nid ensanglanté » dans le Rhésos; mais ces références, qui aboutissent quand même, dans l'article de F. Létoublon, à l'affirmation «peu importe qu'il s'agisse de Philomèle ou de Procné, du rossignol ou de l'hirondelle» (p.95), ne suffisent pas pour oblitérer le fait qu'Ovide dans les Métamorphosess'intéresse moins au paradigme mythologique du chant du rossignol, oiseau du lyrisme endeuillé, qu'à la puissance du modèle exemplaire de la mère infanticide (cf. Médée). Procné-hirondelle devient alors la figure majeure du récit légendaire(deux vers sur trois lui sont consacrés lors de la transformation des deux sœurs en oiseaux). Rompant avec la tradition grecque qui unissait la mère, la mort du fils et la plainte humaine, le poète latin, fidèle à la perspective de son poème décrivant le plus souvent des métamorphoses-punitions, isole Procné sans même valoriser la beauté d'un chant quelconque; la narration aboutit à une tache de sang sur le plumage de l'hirondelle, marque infamante.

76 Les Amours,II, 14, 27-34, déjà nous rappelaient que Procné fut une saeua parens, nous l'avons vu; le ton est plus acerbe dans l'Art d'aimer, II, 383 où figure l'expression dira 
parens. Les Fastes, II, 627-630 excluent de la visite des proches aux dieux de la famille quiconque recourt au crime et englobent dans la même condamnation d'abord Procné puis sa sœur ainsi que Térée. Les deux femmes sont aussi monstrueusement criminelles qu'Atrée, que Médée et qu'Ino. Enfin, les Tristes, III, 12, 9 évoquent le crimen matris et les Pontiques, III, 1, 119-120 stigmatisent l'inpia Progne. Qu'en conclure ? Ovide ne crée pas d'antithèse entre Procné-hirondelle et la monstruosité de la mère infanticide telle Médée: les deux personnages se ressemblent par leur délit horrible et sont des mères funestes. Alors prévaut le droit pénal romain sur toute idée de circonstances atténuantes ; Procné a eu le tort de redoubler l'acte hideux de Térée. Alors que les poètes grecs avaient insisté sur le beau chant du rossignol et en faisaient l'expression du pathos, Ovide distingue le chant et la faute : Procné coupable ne saurait nous émouvoir par la musicalité de sa voix. L'esthétique cède à l'impératif éthique. Seule, l'Héroïde XV semble concilier en Procné-rossignol la plainte et le crime, métamorphosant en beauté la laideur. S'il est vrai que les Métamorphoses figurent un art de la variation par le principe du changement de forme, il convient de ne pas oublier combien certains personnages portent en eux-mêmes cette plasticité significative; ainsi, non seulement Procné et Philomèle sont devenues oiseaux, mais elles ont échangé à la fois leur métamorphose ailée et leur fonction sociale. Seul le jugement négatif sur Procné, épouse transformée en hirondelle, paraît avoir constitué un invariant éthique dans cet univers mobile. Alors Ovide (re)devient sérieux en accentuant le muthos tragique de la violence et de l'horreur; vengeresse de la morale violée, Procné doit être punie à son tour pour avoir cédé à la démesure de la vengeance qui seule, peut-être, a pour légitimité le courroux d'une divinité ; n'oublions pas, en effet, que le livre VI des Métamorphoses où est narrée la triste histoire est aussi celui qui commence par le récit de terribles punitions exercées par des dieux à la colère impitoyable : Arachné, qui a prétendu rivaliser avec Pallas, a été changée en araignée ; Niobé a subi le châtiment de Latone qui a, par ailleurs, changé les paysans lyciens inhospitaliers en grenouilles; enfin le présomptueux Marsyas a été victime d'Apollon. Dans ce contexte, Procné s'est arrogé un droit qui ne lui appartenait pas ; elle a commis, à la différence des divinités en colère, une action illicite qui, parmi les hommes, serait punie d'une peine publique. La variation des tons s'impose donc: ton de compassion, lorsqu'il s'agit de l'exposé et du rappel de faits pitoyables (viol et mutilation de Philomèle, mort d'Itys déplorée par le chant du rossignol qu'est devenue Procné ou Philomèle), ton de condamnation morale pour les faits atroces (adultère de Térée changé en huppe poursuivant sans fin, de sa tête à aigrette et de son long bec en forme de pointe de javelot, son désir de vengeance, infanticide de Procné, hirondelle ensanglantée). Les cris et le chant de ces trois oiseaux différents, s'incarnant en des personnages au statut social nettement défini (époux et père, fille, épouse et mère, fille et sœur), déterminent euxmêmes des changements de tonalité poétique.

Réécrivant en partie le mythe grec, Ovide sut produire par des effets d'intertextualité interne le sentiment de clair-obscur qui nourrit l'interprétation d'une légende simultanément faite de terreur et de pitié.

78 Il reste à chercher, mais cela excède les limites de notre intervention, si le point de vue rigoureusement moral sur Procné - hirondelle a survécu à Ovide. Le mythe, entré dans la catégorie rhétorique des exempla, fournit-il encore de quoi entretenir le rappel de la moralité dans la longue durée de la littérature latine? 


\section{BIBLIOGRAPHIE}

ANDRÉ J., Les noms d'oiseaux en latin, Paris, Klincksieck, 1967.

BRILLANTE Carlo, «Il canto delle pernici in Alcman e le fonti del linguaggio poetico », Revista di Filologia e di Istruzione Classica 119, 1991, p. 150-163

CAPPONI F., Ornitologia latina, Publicazioni dell'instituto di filologia classica e medievale, 58, 1979, Gênes.

D'ARCY W. THOMPSON, A glossary of greek birds, Londres, Oxford University Press, $2^{\circ}$ ed. 1936.

CAZZANIGA I., La saga di Itis nella tradizione letteraria e mitografica greco-romana, Milano, 1950-1951.

DURANGO S., Les oiseaux, Paris, Fernand Nathan.

GÉROUDET P., La vie des oiseaux, Paris - Neuchâtel, Delachaux et Niestlé (6 vol), I : rapaces, colombins, gallinacés, et IV-VI : passereaux.

LÉTOUBLON F., « Le rossignol, l'hirondelle et l'araignée. Comparaison, métaphore et métamorphose », Europe, 904-905, 2004, p.73-102.

LORAUX N., « Le deuil du rossignol », Nouvelle revue de psychanalyse (Paris, Gallimard) 34, 1986, 253-257.

SKODA F., «Contribution à l'étude du lexique animal en grec (formations onomatopéiques, noms imitatifs, emprunts) », in LAMA (Publications du Centre de recherches comparatives sur les langues de la Méditerranée ancienne, Nice), vol. 6 (2), 1974, p. 207-316.

SKODA F., « Principes de formation du lexique animal en grec ancien : illustrations et hypothèses ", in Les Zoonymes, C. Kircher et S. Mellet éds., Publications de la Faculté des Lettres de Nice, vol. 38, 1997, p. 369-386.

SPATAFORA G., « Il pianto dell'usignolo nelle poesia greca antica », Orpheus 16 (1), 1995, p. 98-110.

ZAGANIARIS N.J., « Le mythe de Térée dans la littérature latine et grecque », Platon 25,1973, p. 208-23

\section{NOTES}

1. Voir par exemple Photios, Bibliothèque 186, 136a 29 [Bekker].

2. «Pandion épousa la sœur de sa mère, Zeuxippè, et engendra deux filles, Procné et Philomèle, et deux fils jumeaux, Erechtheus et Boutès. Une guerre ayant éclaté avec Labdacos pour une question de frontières, il appela de Thrace à son secours Térée, fils d'Arès, et lorsque, avec son aide, il eut terminé la guerre avec succès, il donna en mariage à Térée sa fille Procnè. Térée eut d'elle un fils, Itys, mais il s'éprit de Philomèle aussi et la viola. Lui disant que Procnè était morte, il la cacha à la campagne et continua [après l'avoir épousée] à coucher avec elle. Il lui coupa la langue. Mais elle tissa un message dans une étoffe et elle parvient ainsi à prévenir Procnè de ses malheurs. Celle-ci, après avoir retrouvé sa sœur, tue son fils, Itys, le fait cuire et le sert comme repas à Térée, qui ne se doute de rien. Puis elle s'enfuit en hâte avec sa sœur. Quand Térée eut compris, il se saisit d'une hache et se lança à leur poursuite. Lorsque, arrivées à Daulis de Phocide, elles se voient rejointes, elle demandent aux dieux de les changer en oiseaux. Procné 
devient un rossignol et Philomèle une hirondelle. Térée aussi est changé en oiseau et il devient une huppe ». (Apollodore, Bibliothèque III, 193-195, trad. J.-Cl. Carrière et B. Massonie, 1991, Annales littéraires de l'Université de Besançon).

3. Les métamorphoses, 11 " Aédon".

4. Odyssée 19, 518-523 : «Comme quand la fille de Pandarée, la chanteuse verdière ,/ chante un beau chant au premier retour du printemps, / posée dans le feuillage innombrable des arbres / et modulant souvent, elle file sa voix très douce, / pleurant sur Itylos, son fils, qu'elle a tué, / par méprise d'un coup d'épée, fils du seigneur Zéthos ». (traduction de P. Jaccottet). Scholie à Odyssée 19, 518 : «Zêthos épouse Aêdon fille de Pandareos, et il leur naît Itylos et Nêis. Aêdon tue son fils Itylos, une nuit, le prenant pour le fils d'Amphion, jalouse qu'elle est de la mère de celui-ci, parce qu'elle a six enfants, alors qu'elle-même n'en a que deux. Zeus la condamne à un châtiment et elle le prie de la transformer en oiseau ; Zeus fait d'elle un rossignol ; et elle se lamente toujours sur Itylos, comme le dit Phérécyde ».

5. Eustathe (Commentarii ad Homeri Odysseam, vol. 2, p 215) distingue bien la légende thébaine et la légende attique.

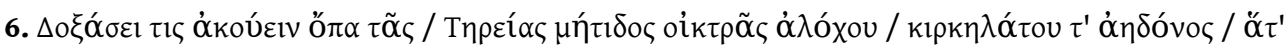

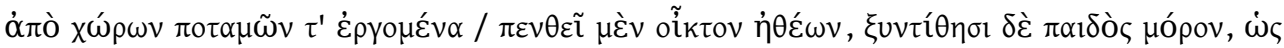

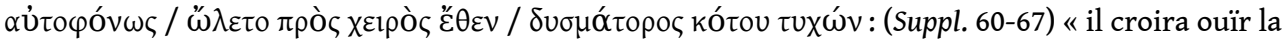
voix de l'épouse de Térée, pitoyable en ses remords, la voix du rossignol que poursuit un oiseau de proie. Chassée de son séjour d'antan, elle pleure douloureusement sa demeure familière, tout en disant la mort de son enfant, comment il succomba sous sa main, sous ses propres coups,

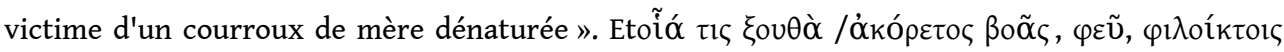

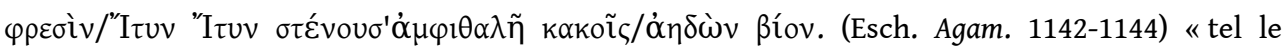
rossignol fauve, jamais las d'appeler 'Itys! Itys!' gémit, hélas! en son cœur douloureux sur une vie trop riche de douleurs ".

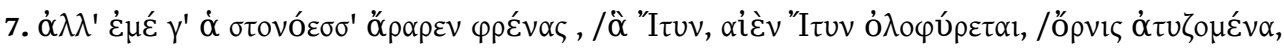
$\Delta$ ò̀ $\alpha^{\alpha} \gamma \gamma \varepsilon \lambda$ oc. (Soph. Elec. 147-149) «ce qui répond à mon humeur, à moi, c'est l'oiseau qui se lamente en répétant 'Itys! Itys!', c'est l'oiseau désespéré qui sert de messager à Zeus ».

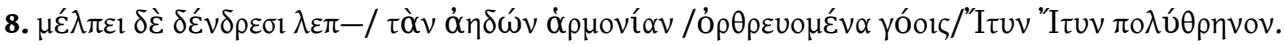
(Eur. Phaeton, fr. 23, 68-70 [J. Diggle, 1970]) = fr. 773, 21-25 [Nauck]) « Dans les arbres, le rossignol exhale les harmonies de son chant subtil. Eveillé dès l'aurore, il sanglote sur Itys, Itys mille fois pleuré ».

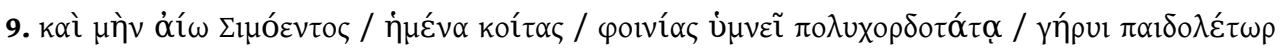

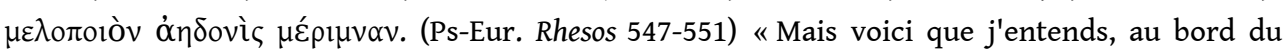
Simoïs, perché sur son nid ensanglanté, chanter le rossignol meurtrier de son enfant : il exhale sa plainte en trilles mélodieux $»$.

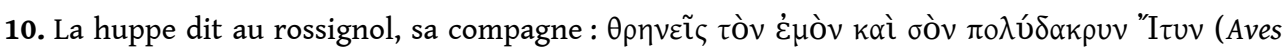
211-212) « tu déplores l'objet pour toi et pour moi de tant de larmes, notre Itys ».

11. V, 3, 3-8.

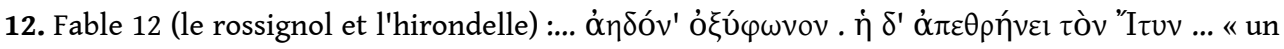
rossignol à la voix aiguë ; il déplore la mort d'Itys ».

13. Dionysiaca 2, 131-139: parallèle entre une nymphe qui craint la violence amoureuse de Typhée et Philomèle, l'hirondelle, qui a subi les violences de Térée.

14. Libanius, Progymnasma 2, 19, 1 : «Térée prit pour épouse Procné d'Athènes, fille de Pandion. [...] Térée, ayant emmené Philomèle, se mit à la désirer en chemin, et lui fit violence à cause de ce désir et, après l'avoir violentée, lui déroba le moyen de le confondre en lui coupant la langue. Celle-ci, arrivée chez Procné, ne pouvait pas dire sa souffrance, mais en tissant une toile, elle raconte avec sa main ce qui lui était arrivé. En apprenant ce que sa sœur avait subi, Procné offre 
son enfant en nourriture à son père " [suit le récit de la poursuite et l'évocation de la métamorphose].

15. Les seuls textes grecs qui, à ma connaissance, contreviennent à cette répartition sont tardifs : ce sont le récit fait par Eustathe (Commentarii ad Homeri Odysseam, Vol. 2, p. 215) et une scholie aux Halieutiques d'Oppien : « du rossignol : Procné et Philomèle étaient sœurs : Philomèle habitait avec son époux, et avait un enfant ; le mari de celle-ci alla avec Procné. L'apprenant, Philomèle tue son enfant » (Schol. in Oppianum, Schol. et glossae in Halieutica, scholion 728).

16. Le a final est long (ion. $-\eta, \eta \varsigma$ ).

17. Cf. F. Bechtel, Die histor. Personennamen des Griechischen (Halle, 1917, repr. 1964), p. 315 :

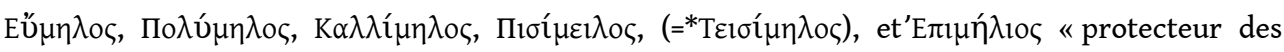
moutons ", qui est une épithète d'Apollon et d'Hermès.

18. Théocr. Buc. IV 16, Call. Ap. 41.

19. Comme l'ont proposé bien des traducteurs, et le rédacteur de l'article du LSJ (« dusky, dark in colour »).

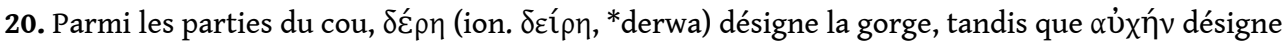

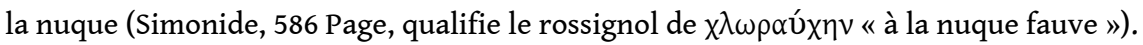

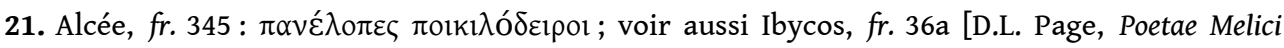

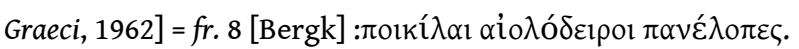

22. P. Vasak, Oiseaux des forêts, Gründ, 1992, p.150-151.

23. Voir aussi Eustathe, Commentarii ad Homeri Odysseam, vol. 2, p. 215 (cité supra).

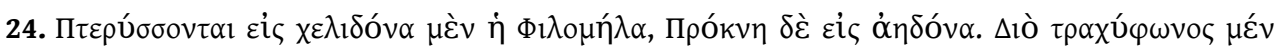

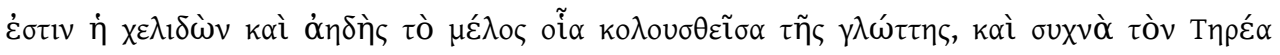

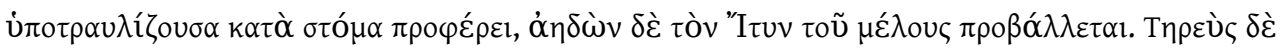

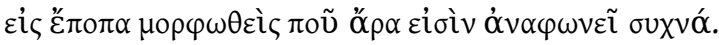

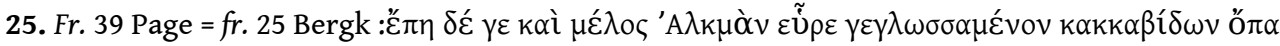

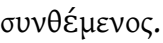

26. La perdrix n'a pas été choisie au hasard: elle a une grande diversité de rythmes de chants, auxquels réfèrent en français au moins une demi-douzaine de verbes différents : elle glousse, rappelle, cacabe, brouit, pirouitte... Les attagas d'Elien sont probablement aussi une espèce de perdrix.

27. Hésychius, $\mathrm{t} 517$.

28. Hésychius t 993, Suda t 695, Babrios, 2, 131, 8 .

29. Esch. Agam. 1142-1142, Soph. Elec. 148.

30. P. Oxy. 2625, fr. 1, 7sq. Voir I. Rutherford, «The nightingale's refrain : P. Oxy. $2625=$ SLG 460 », Z.P.E. 107,1995 , p. 39-43.

31. Fr. 297 Nauck. Bien qu'Aristote attribue ce fragment à Eschyle, certains chercheurs le pensent extrait du Térée de Sophocle (de 427), ou de celui de Philoclès (de 426), l'existence de la tétralogie de ce dernier sur les filles de Pandion n'étant connue que par une Scholie à Oiseaux 281.

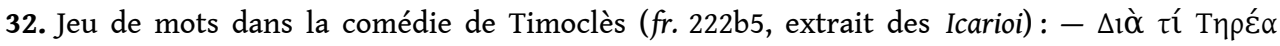

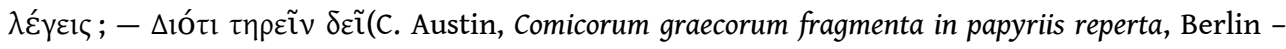
New-York, W de G, 1973, p. 218).

33. "Dès l'arrivée des nicheurs, et jusqu'au moment où les nichées sont en train, le mâle émet son houpoupoup de trois syllabes, parfois de deux, rarement de quatre; chaque fois il baisse la tête, le bec, fermé ou presque, pointant vers le sol, puis le relève à la fin de la strophe ; ce chant doux et monotone, assez bas et sourd, n'est pas sonore de près, mais porte fort loin » (P. Géroudet, La vie des oiseaux, IV, 61-67).

34. « Elles » pour la scholie à Hésiode citée plus haut.

35. Ce signifié se dit koũ dans l'ionien d'Hérodote. 


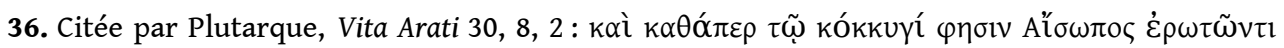

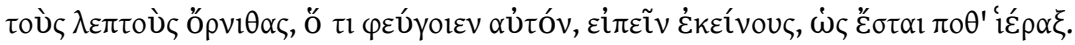

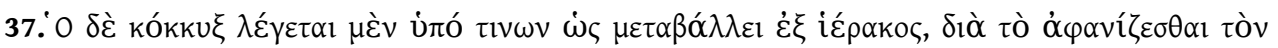

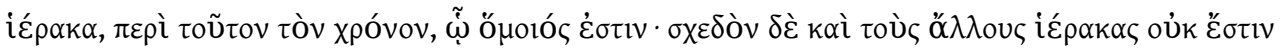

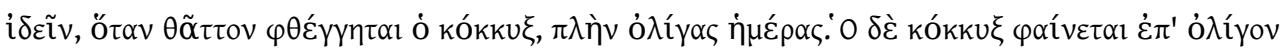

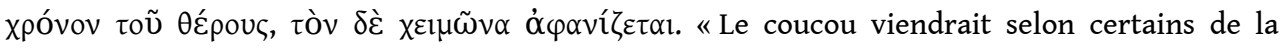
métamorphose d'un hierax, parce que le hierax auquel il ressemble disparaît au moment où luimême fait son apparition. En fait, il est presque aussi exceptionnel de voir les autres espèces de hierax dès que le coucou se fait entendre, sauf pendant quelques jours. Le coucou ne se montre que peu de temps pendant la saison chaude, et il disparaît pendant l'hiver ».

38. C'est le cas dans la Fable 45 d'Hygin (qui par ailleurs assimile son épouse Procné à l'hirondelle, et Philomèle au rossignol): Tereus Martis filius Thrax cum Prognen Pandionis filiam in coniugium haberet, [...]Progne autem filium Itym ex se et Tereo natum occidit, patrique in epulis apposuit et cum sorore profugit. Tereus facinore cognito fugientes cum insequeretur, deorum misericordia factum est ut Progne in hirundinem commutaretur, Philomela in lusciniam; Tereum autem accipitrem factum dicunt.

39. Le kirkos est le moins puissant des hierax (Arstt. H.A. 620 a, 18).

40. Le mot épervier lui-même vient du germanique Sparvari, dérivé de Spar « moineau ».

41. Du latin passer « passereau, moineau ».

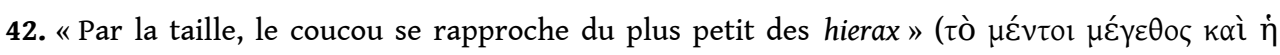

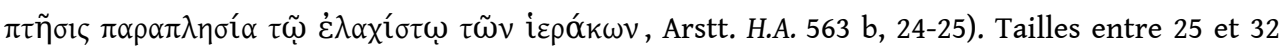
$\mathrm{cm}$, envergures entre 55 et $70 \mathrm{~cm}$.

43. Sophocle (E. Col. 671) qualifie ainsi d'aiguë ( $\lambda$ í $\varepsilon \varepsilon \alpha)$ la voix du rossignol.

44. Ainsi les noms de l'hirondelle et du rossignol sont au féminin, et ceux du coucou, de la huppe et de l'épervier au masculin aussi bien en grec et en latin qu'en allemand.

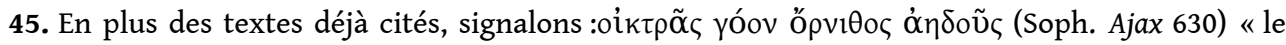

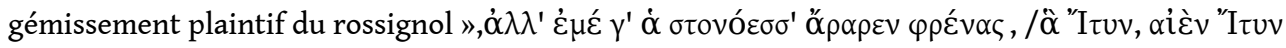

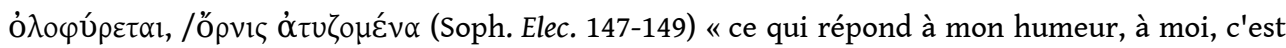

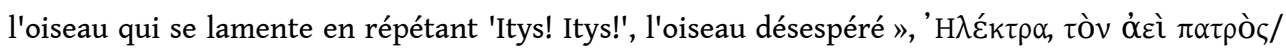

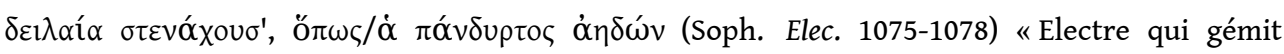
perpétuellement le deuil de son père, la malheureuse, comme le plaintif rossignol ", 'Aүxoṽ $\delta^{\prime}$

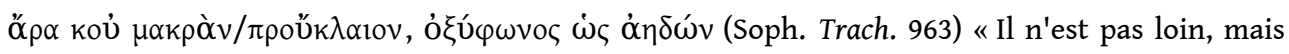
tout près au contraire, le malheur que j'étais à déplorer d'avance avec la voix aiguë du rossignol

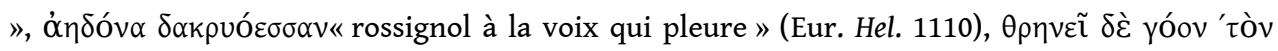

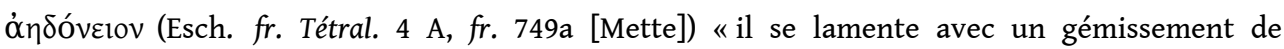
rossignol». Voir aussi l'étude de D. Arnould au chapitre 12 («l'oiseau, symbole littéraire des pleurs ») de Le rire et les larmes dans la littérature grecque d'Homère à Platon, Paris, les Belles Lettres, 1990.

46. Anthologie Palatine IX 57,$70 ; X 4,5$.

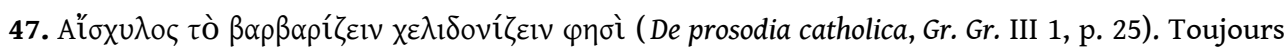
d'après le même passage d'Hérodien, «Ion, dans l'Omphale, appelle les Barbares hirondelles, en

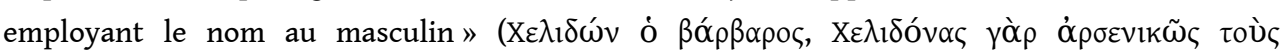

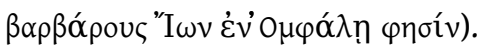

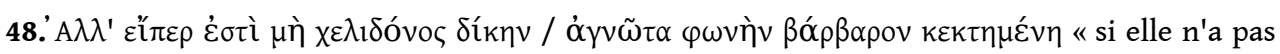
un langage inconnu de barbare, comme l'hirondelle... ».Voir aussi Ar. Gren. 680-683.

49. Cette dernière observation serait confortée si le mythe n'a pas de parallèles dans les autres cultures d'origine indo-européenne. Personne n'a jamais signalé de parallèle avec le trio d'oiseaux du mythe grec. Dans différentes littératures européennes, il y a des fables de rossignols persécutés par un épervier (Hésiode) ou un rapace nocturne (Chaucer, The Owl and the Nightingale 
), ou de rossignol mélodieux opposé à un coucou ou un hibou qui criaille. On trouve aussi des éperviers poursuivant des hirondelles ou d'autres petits oiseaux. Mais, à notre connaissance, pas de trio coucou - rossignol - hirondelle, ni chouette - rossignol - hirondelle (la chouette, ou le grand duc, sont des oiseaux qui disent « où? » en français, et « wo? » en allemand).

50. Et manibus Procne pectus signata cruentis $<\mathrm{Qu}$ 'on éloigne> Procné à la poitrine marquée de ses mains ensanglantées.

51. Coniugis admissum uiolataque iura marita est / Barbara per natos Phasias ulta suos; / Altera dira parens haec est, quam cernis, hirundo; / Adspice, signatum sanguine pectus habet. «Le crime d'un époux, la violation de la loi conjugale, une épouse barbare, née aux bords du Phase, les vengea sur ses enfants. Une autre mère dénaturée, c'est cette hirondelle que tu vois. Regarde-la, elle a encore du sang sur sa poitrine » (C.U.F., 1983, H. Bornecque).

52. Tantalidae fratres absint et Iasonis uxor / Et quae ruricolis semina tosta dedit / Et soror et Procne Tereusque duabus iniquus / Et quicumque suas per scelus auget opes. «Ici sont indésirables les deux frères, petits-fils de Tantale, l'épouse de Jason, celle qui remit aux paysans des semences grillées, Procné et sa sœur ainsi que Térée, leur bourreau à toutes deux, enfin quiconque recourt au crime pour augmenter sa fortune » (C.U.F., 1992, R. Schilling).

53. Fallimur, an ueris praenuntia uenit hirundo / Et metuit ne qua uersa recurrat hiems? / Saepe tamen, Procne, nimium properasse quereris / Virque tuo Tereus frigore laetus erit « Me trompè-je ou le printemps s'annonce-t-il par l'arrivée de l'hirondelle, même si elle redoute que l'hiver ne fasse retour? Souvent, pourtant, tu te plaindras, Procné, d'avoir mis trop de hâte et ton époux Térée se réjouira que tu aies froid » (C.U.F., 1992, R. Schilling).

54. Utque malae matris crimen deponat hirundo, / Sub trabibus cunas tectaque parua facit, « (...) pour se laver du crime de sa (?) méchante mère, l'hirondelle bâtit sous les poutres la petite demeure de son nid » <nous proposons plutôt la traduction : pour se laver de son crime de méchante mère (...)> (C.U.F., 1968, J. André).

55. Flet Philomela nefas incesti Tereos, et quae / Muta puella fuit, garrula fertur auis. « Philomèle pleure sur le crime de l'incestueux Térée; elle fut une fille muette, elle est célèbre comme oiseau chanteur » (C.U.F., troisième édition, 1973, H. J. Izaac).

56. Aut ut mutatis Terei narrauerit artus, / quas illi Philomela dapes, quae dona pararit, / quo cursu deserta petiuerit, et quibus ante / infelix sua tecta super uolitauerit alis? « Ou rappellerai-je comment il a narré la métamorphose de Térée, les mets, les présents que Philomèle lui a préparés, sa fuite vers les solitudes, et les ailes qui permirent d'abord à la malheureuse de survoler son toit! » (Classiques en Poche, E. de Saint-Denis/J.-P. Néraudau, Les Belles-Lettres, 2001).

57. Qualis populea maerens Philomela sub umbra / amissos queritur fetus, quos durus arator / obseruans nido implumis detraxit; at illa / flet noctem, ramoque sedens miserabile carmen / integrat et maestis late loca questibus implet. Telle la triste Philomèle, sous l'ombre du peuplier, regrette ses petits perdus qu'un paysan insensible aux aguets a tirés sans plumes de leur nid. Et elle, elle pleure la nuit et posée sur la branche, elle reprend son chant de douleur, au loin elle remplit les lieux de ses tristes plaintes.

58. Quod scelus Ismarii quereris, Philomela, tyranni, / Expleta est annis ista querela tuis ; / Alitis in rarae miserum deuertere funus; / Magna, sed antiqua est causa doloris Itys « Pourquoi, Philomèle, gémir sur le crime du tyran ismarien? Avec les années, tu dois être lasse de gémir. Reporte-les sur la triste mort d'un oiseau rare. C'est un grand sujet de douleur qu'Itys, mais un sujet bien lointain » (C.U.F., 1968, H. Bornecque).

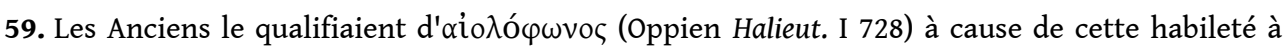
apporter des changements à sa voix.

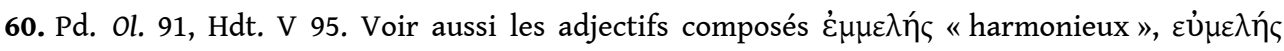
« mélodieux ».

61. Colchida respersam puerorum sanguine culpant / Atque sua caesum matre queruntur Ityn; / Utraque saeua parens: sed tristibus utraque causis / Iactura socii sanguinis ulta uirum. « On maudit la fille du 
roi de Colchide souillée du sang de ses enfants et l'on plaint Itys égorgé par sa mère. Toutes deux furent des mères cruelles ; mais toutes deux eurent de graves motifs pour se venger de leur mari sur leur postérité commune. » (C.U.F., 1968, H. Bornecque).

62. Concinit Odrysium Cecropis ales Ityn « L'oiseau athénien chante l'Odrysien Itys. » (C.U.F., 1968, H. Bornecque).

63. Alloquar, audiero numquam tua facta loquentem, / Numquam ego te, uita frater amabilior, / Aspiciam posthac ; at certe semper amabo, / Semper maesta tua carmina morte tegam, / Qualia sub densis ramorum concinit umbris / Daulias absumpti fata gemens Itylei. «J'aurai beau t'adresser la parole, jamais plus je ne t'entendrai parler de ce que tu as fait, jamais plus, ô mon frère, qui m'étais plus cher que la vie, je ne te verrai désormais ; mais du moins je t'aimerai toujours; toujours je composerai dans la retraite des chants attristés par ta perte, semblables aux gémissements que fait entendre sous les épais ombrages la Daulienne, déplorant la cruelle mort d'Ityle. » (C.U.F., 1974, G. Lafaye).

64. Alcyonum positis requiescant ora querelis ; / increpet absumptum nec sua mater Ityn. " Puissent les alcyons faire taire leurs cris plaintifs, leurs gosiers retrouver la paix, et que la mère d'Itys ne pleure plus son fils disparu. » (Imprimerie Nationale, Editions, 2003, P. Charvet).

65. Sola uirum non ulta pie maestissima mater / Concinit Ismarium Daulias ales Ityn. « (...) seul l'oiseau de Daulis, mère douloureuse qui tira de son époux une vengeance impie, chante Itys l'Ismarien. » (C.U.F., 1999, H. Bornecque/D. Porte).

66. Quacumque ingreditur, miseris loca cuncta querelis / inplet, ut amissum cum gemit ales Ityn «Partout où elle porte ses pas, elle emplit tous les lieux de ses plaintes pitoyables, comme fait l'oiseau qui pleure son Itys perdu » (éd. Pàtron, Bologne, 1970, H. Le Bonniec).

67. Tingeret ut ferrum natorum sanguine mater, / Concitus a laeso fecit amore dolor. / Fecit amor subitas uolucres cum paelice regem / Quaeque suum luget nunc quoque mater Ityn. «Le désespoir d'un amour outragé poussa une mère à teindre un fer du sang de ses enfants. L'amour fit métamorphoser soudain en oiseaux un roi et sa maîtresse, et une mère qui maintenant encore pleure son cher Itys. » (C.U.F., 1968, J. André).

68. Est aliquid fatale malum per uerba leuare. / Hoc querulam Procnen Alcyonemque facit « C'est quelque chose d'alléger par des mots le destin qui nous frappe. C'est pourquoi gémissent Procné et Alcyoné. » (C.U.F., 1968, J. André).

69. Quid trepidas et adire times? Non inpia Progne / filiaue Aeetae uoce monenda tua est, "Pourquoi trembles-tu et crains-tu de l'approcher? Ce n'est pas l'impie Progné ou la fille d'Aéétès que ta voix doit émouvoir (...) »(C.U.F., 1977, J. André).

\section{RÉSUMÉS}

Térée, Procné et Philomèle : du mythe aitiologique au début du mythe littéraire

Les variantes et les gloses du mythe dans les textes grecs sont autant d'indices pour montrer qu'il a pour fondement une fable animalière: l'interaction de trois oiseaux aux noms étymologiquement signifiants (Procné, la "tachetée ", est un rossignol, Philomèle, "l'amie des troupeaux", une hirondelle, Térée, le «guetteur", une huppe plus anciennement à la fois épervier et coucou), dont le comportement animal, attentivement observé, a été interprété sur le modèle des relations sociales et subjectives entre êtres humains et dans le cadre d'homologies entre le cri de chaque oiseau et un mot du langage humain. L'interaction familiale et passionnelle l'a ensuite emporté sur la composante animalière, créant l'état grec du mythe où les personnages 
ne sont plus animaux que lors de leur métamorphose finale. Puis, en terre italienne, c'est l'observation d'hirondelles et de rossignols d'autres espèces qui a conduit aux échanges, bien connus mais déconcertants, de noms et de rôles des deux femmes. Le mythe y a gagné une plasticité qui a permis aux poètes latins de le remodéliser de multiples façons, selon la facette morale ou esthétique qu'ils souhaitaient mettre en valeur.

Tereus, Procne and Philomele : from the aitiological myth to the beginning of the literary myth Variants and glosses of this myth in Greek texts are clues indicating that it is based upon an ancient fable about animals: the interaction of three birds whose names have a significant etymology (Procne, "speckled", is a sort of nightingale, Philomele, "friend of flock", is a sort of swallow, Tereus, "watchman", is a hoopoe that was, in elder time, both a hawk and a cuckoo) and whose animal behavior, carefully observed, has been interpreted on the pattern of social and subjective relations between men and with homologies between the cry of each bird and a word of human language. Next, the familial and impassioned interaction has prevailed over the animal component, creating the situation of the Greek myth in which the heroes are birds only when they are metamorphosed. Then, on Italian land, it was through the observation of other sorts of nightingales and swallows that people were led to exchange names and parts of the two sisters. The myth has then gained a plasticity that allowed Latin poets to modify it in many ways, according to the moral or aesthetic facet they wanted to emphasize.

\section{AUTEURS}

\section{MICHĖLE BIRAUD}

Université de Nice. BCL (Bases, Corpus, Langage). Linguistique grecque.

biraud@unice.fr

\section{EVRARD DELBEY}

Université de Nice 